\title{
Aço carbono 1040 como indicador de agressividade do solo e da pasta de cimento em sistemas de ancoragem de linhas de transmissão
}

\author{
Carbon steel 1040 as an indicator of the \\ aggressiveness of soil and cement paste \\ in anchorage systems of transmission lines
}

\author{
Tiago Scheffer de Matos ${ }^{1,2}$, Kleber Franke Portella ${ }^{1,2}$ \\ Sérgio Luiz Henke ${ }^{3}$, Mariana D’Orey Gaivão Portella Bragança ${ }^{1}$ \\ Bruno Endo Ribeiro ${ }^{1}$, Igor Miguel Pinkoski ${ }^{1}$ \\ Alessandro Cesar de Sousa Berrêdo ${ }^{4}$
}

\footnotetext{
${ }^{1}$ Institutos Lactec - Rodovia BR-116, km 98, no 8.813, Jardim das Américas, CEP: 81531-980, Curitiba, Paraná, Brasil ${ }^{2}$ Universidade Federal do Paraná, Programa de Pós Graduação em Engenharia e Ciências dos Materiais-PIPE, Curitiba, Paraná, Brasil.

${ }^{3}$ Universidade Federal do Paraná, Departamento de Engenharia Mecânica, Curitiba, Paraná, Brasil.

${ }^{4}$ Transmissora Aliança de Energia Elétrica S.A. -TAESA, Praça Quinze de novembro, Centro, Rio de Janeiro, Rio de Janeiro, Brasil.

e-mail: tsmatosqui@yahoo.com.br, portella@lactec.org.br, henke@ufpr.br, mariana.portella@lactec.org.br, bruno.ribeiro@lactec.org.br, igor.pinkoski@lactec.org.br, alessandro.berredo@taesa.com.br
}

\section{RESUMO}

O encapsulamento de hastes de âncora em pasta de cimento reduz o contato dos materiais metálicos com os ambientes agressivos, em torres estaiadas de linhas de transmissão de energia elétrica. Contudo, ainda são reportados casos de falha prematura devido à corrosão do sistema de ancoragem. $\mathrm{O}$ íon cloreto é considerado um dos principais responsáveis pelo início e pela propagação da corrosão. Entretanto, existem muitas divergências em relação a concentração crítica de cloreto no solo e na pasta de cimento e a sua influência na formação dos produtos de corrosão. Neste estudo, o aço carbono 1040 foi exposto em laboratório a ambientes com diferentes concentrações de íons cloreto, 764 e $1764 \mathrm{mg} / \mathrm{kg}$ no solo, 2,0\% e 3,5\% na pasta de cimento, teores estes, baseados nos resultados analíticos obtidos em amostras de hastes e de seu entorno, retiradas do campo. A investigação do estado quanto à corrosão do metal em laboratório foi efetuada ao longo do tempo, pela medida do potencial de circuito aberto (OCP- "Open Circuit Potential") e pela espectroscopia de impedância eletroquímica (EIE). Os produtos de corrosão foram caracterizados por microscopia eletrônica de varredura, com análise química elementar (MEV/EDS) e difração de raios-X (DRX). Por meio da análise de EIE foi possível detectar a evolução do processo corrosivo do metal no solo e na pasta de cimento, mesmo sendo observadas diferentes formas de corrosão: generalizada no primeiro e localizada nesta última, respectivamente. $\mathrm{Na}$ avaliação dos produtos de corrosão, por MEV e DRX, foi inferida a presença de goetita, e calcita na superfície do aço exposto ao solo, e no encapsulado em pasta cimento foi possível visualizar os pites formados e as composições do seu entorno superficial. Esses resultados indicaram a necessidade da utilização de metais mais resistentes à corrosão em ambientes com íons cloreto, visando o aumento da durabilidade do sistema de ancoragem.

Palavras-chave: hastes de âncora, corrosão, cloretos, solo, pasta de cimento.

\section{ABSTRACT}

The encasement of anchor rods in cement paste reduces the contact of metal materials with aggressive environments in guyed power transmission line towers, however, many cases of premature failure due to corrosion of the anchorage systems are still reported. Chloride ions are considered to be one of the main factors responsible for the initiation and propagation of corrosion, however, there are many differences regarding the critical concentration of chloride in the soil and cement paste and their influence on the formation of corro- 
sion products. In this study carbon steel 1040 was exposed in the laboratory to environments with different concentrations of chloride ions, 764 and $1764 \mathrm{mg} / \mathrm{kg}$ in the soil, $2.0 \%$ and 3.5\% in the cement paste, based on the analytical results obtained in samples of rods and their surroundings, taken from the field. The investigation of the corrosion state of the metal in the laboratory was evaluated, over time, by the measurement of open circuit potential (OCP), electrochemical impedance spectroscopy (EIS). The corrosion products were characterized by scanning electron microscopy with elemental chemical analysis (SEM/EDS) and X-ray diffraction (XRD). By means of the EIS analysis, it was possible to detect the evolution of the corrosive process of the metal in the soil and cement paste, even though different forms of corrosion were observed: general in the soil and localized in the cement paste. Moreover, the evaluation of the corrosion products, by SEM and $\mathrm{XRD}$, indicated the presence of goethite and calcite on the steel surface exposed to the soil, and in the steel encapsulated in cement paste it was possible to visualize the pits formed and the compositions of their surface surroundings. These results suggest to need to use corrosion resistant metals in environments with chloride ions, in order to increase the durability of the anchorage system.

Keywords: anchor rods, corrosion, chloride, soil, cement paste.

\section{INTRODUÇÃO}

A utilização de torres estaiadas, ancoradas por hastes metálicas e que são fixadas diretamente ao solo ou encapsuladas em pasta de cimento, é um processo comum na construção de linhas de transmissão de energia elétrica (LT), conforme mostrado na Figura 1 (a) e (b). Entretanto, a corrosão de suas hastes (Figura 1 (b)), vem ocasionando problemas de segurança e de confiabilidade, uma vez que a mesma pode causar a queda de parte da LT, pelo seu rompimento, além de causar um impacto econômico devido à redução da vida útil do sistema de ancoragem [1,2].
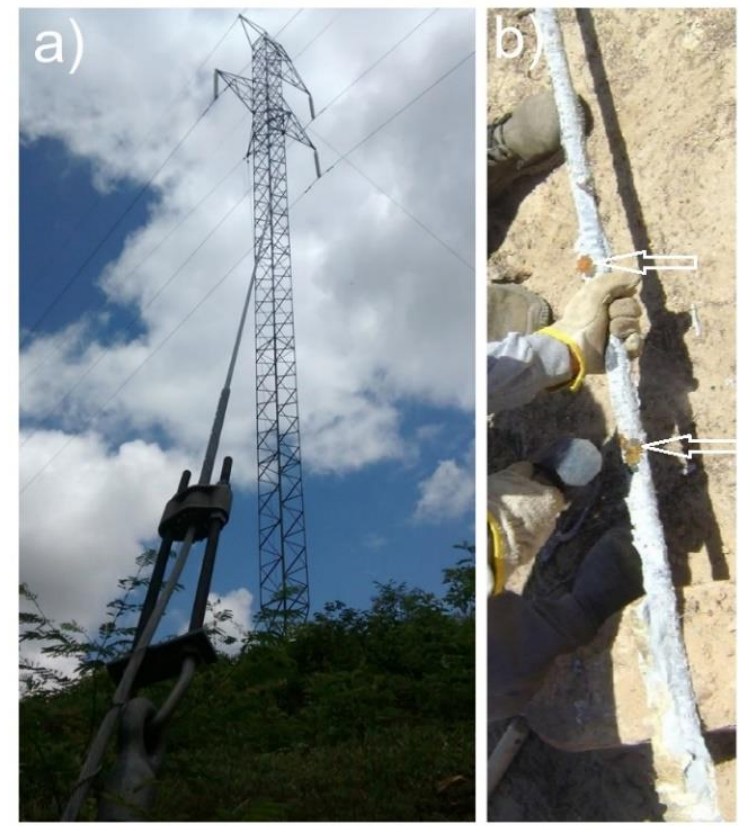

Figura 1: Fotos ilustrativas: (a) de uma das torres estaiadas de uma linha de transmissão de energia elétrica instalada no nordeste do Brasil; (b) do problema de corrosão em determinadas regiões (setas) de uma das hastes enterradas por cerca de 10 anos.

Recentes estudos têm apontado para diversos fatores que podem influenciar na corrosão dos materiais metálicos em solo, incluindo a umidade, o oxigênio, o teor de sais dissolvidos, a atividade microbiológica e outros $[3,4,5]$. Esse grande número de variáveis ocasiona dificuldades na identificação e na compreensão do mecanismo e da influência de cada parâmetro na taxa de corrosão[6].

A obtenção da estrutura e da composição dos produtos de corrosão do aço carbono e da sua correlação com o processo de dissolução do metal é uma tarefa complexa devido a transformação desses compostos com o passar do tempo, além da influência dos fatores ambientais de exposição, como a presença de íons que podem alterar o mecanismo de corrosão [7]. Porém, vários estudos têm demostrado avanços significativos no reconhecimento das características morfológicas dos compostos formados durante a corrosão do aço carbono, 
seja pela síntese desses compostos em laboratório ou pela análise de informações de técnicas de caracterização $[8,9,10]$.

Nesse âmbito Duffó et al. [11] investigaram a formação de produtos de corrosão no aço carbono encapsulado no concreto e evidenciaram a formação de uma camada compacta e aderente de magnetita $\left(\mathrm{Fe}_{3} \mathrm{O}_{4}\right)$ junto a superfície do metal, e uma camada porosa e não aderente formada principalmente por lepidocrocita $(\gamma-\mathrm{FeOOH})$ e goethita $(\alpha-\mathrm{FeOOH})$ na parte externa dos produtos de corrosão.

$\mathrm{O}$ encapsulamento dos materiais metálicos em pasta de cimento foi apontado como uma alternativa de proteção, pois forma uma barreira física contra os agentes externos que podem acelerar a corrosão. Além disso, a elevação do pH do meio, devido às reações de hidratação do cimento, induz a passivação do aço carbono segundo diagrama de Pourbaix [12]. Entretanto, com o passar o tempo, o ingresso de íons, como cloreto e sulfato, e o processo de carbonatação, levam a redução do pH, e induzem a saída do seu estado passivo[13].

A barreira física que a pasta de cimento propicia ao aço, muitas vezes, não é suficiente para impedir a entrada de sais dissolvidos. Esses sais ingressam nos poros da pasta de cimento, e são transportados pelos caminhos condutivos preferenciais, atingindo a interface com o metal, onde interagem não só com este, como também com a própria matriz cimentícia, o que pode contribuir para a redução do $\mathrm{pH}$ e consequentemente para o aumento da taxa de corrosão do aço [14,15,16].

A investigação dos processos corrosivos em metais utilizados em sistemas de ancoragem tem sido um dos grandes desafios dos pesquisadores nas últimas décadas, especialmente em meios resistivos como o solo e pasta de cimento, além disso, em algumas das técnicas eletroquímica disponíveis, é necessário a aplicação de um potencial ou corrente que altera ou induz a corrosão no metal, como no caso do método de extrapolação de Tafel, onde os sobrepotenciais aplicados acabam modificando a superfície do metal. [17,18].

A espectroscopia de impedância eletroquímica (EIE) é considerada uma técnica não destrutiva de análise, ou seja, os baixos valores de sobrepotenciais aplicados na forma senoidal (aprox. 10-25 mV) em relação ao estado de repouso do sistema de medida, praticamente não provocam alterações no metal em estudo. A EIE tem sido muito utilizada na avaliação e na interpretação de fenômenos físico-químicos, elétricos e mecânicos que acontecem nas interfaces e no interior de diversos materiais [19, 20].

Em um estudo desenvolvido por SANTOS et al. [21], a EIE foi utilizada em conjunto com medidas de potencial de circuito aberto e curvas de polarização, na investigação a corrosão causada pela polpa de bauxita em um mineroduto do aço carbono, onde foi constatado por meio dos diagramas de EIE a redução da resistência a polarização do aço carbono em contato com a polpa de bauxita em relação a água como eletrólito, além disso esses resultados foram corroborados pelos ensaios de polarização realizados pelo autor.

Neste trabalho, foi avaliada a suscetibilidade à corrosão do aço carbono imerso em solo e em pasta de cimento durante um período de 75 dias por meio da técnica EIE, em conjunto com a análise morfológica e de composição dos produtos de corrosão formados por microscopia eletrônica de varredura (MEV/EDS) e DRX.

\section{MATERIAIS E MÉTODOS}

\subsection{Materiais}

Hastes de aço carbono 1040, em formato cilíndrico, com $8 \mathrm{~cm}$ de comprimento e $1 \mathrm{~cm}$ de diâmetro, foram utilizadas nos ensaios de corrosão em solo e em pasta de cimento, assim como nos ensaios eletroquímicos. A sua composição química elementar pode ser visualizada na Tabela 1.

Tabela 1: Composição química do aço carbono 1040, utilizado nos ensaios de corrosão em solo e em pasta de cimento.

\begin{tabular}{c|c|c|c|c|c|c}
\hline \multicolumn{7}{c}{ ELEMENTOS (\% MASSA) } \\
\hline & $\mathrm{C}$ & $\mathrm{Si}$ & $\mathrm{Mn}$ & $\mathrm{P}$ & $\mathrm{S}$ & $\mathrm{Fe}$ \\
\hline Haste analisada & 0,47 & 0,25 & 0,66 & 0,022 & 0,012 & balanço \\
\hline
\end{tabular}

O solo utilizado na análise do desempenho do metal quanto ao processo de corrosão foi coletado em uma região próxima a uma torre estaiada de uma linha de transmissão de energia elétrica (LT) no Nordeste do Brasil, em estudo de caso. Este solo contém um teor de cloreto médio de $(764 \pm 12) \mathrm{mg} / \mathrm{kg}$, sendo este, denominado de solo 01 .

Para se ter um indicativo da influência do teor de cloreto no desempenho do metal no meio e na for- 
mação dos produtos de corrosão, foi confeccionada mais uma amostra, esta denominada solo 02, na qual foram adicionados, em uma porção do solo $01,1000 \mathrm{mg} / \mathrm{kg}$ de cloreto, uma vez que teores similares deste contaminante foram também encontrados em outras regiões da LT em análise, tanto no solo quanto na pasta de cimento [22], cuja extensão ultrapassa $200 \mathrm{~km}$ lineares, em território nordestino.

As composições químicas de íons livres, tanto na pasta de cimento quanto no solo, foram obtidas a partir da sua solubilização e posterior análise em um equipamento Cromatógrafo de Íons, marca Metrohm, modelo 881 Compact IC Pro, com coluna cromatográfica Metrosep A Supp 5-150/4.0, sistema de supressão química e detector de condutividade nas condições de eluição de $0,7 \mathrm{~mL} / \mathrm{min}$ utilizando como eluente uma solução de $\mathrm{Na}_{2} \mathrm{CO}_{3} / \mathrm{NaHCO}_{3}$ com concentrações de 5,0 e 1,0 mmol/L respectivamente. Ambas as amostras de solo tiveram o $\mathrm{pH}$ de $9,42 \pm 0,05$.

Outras amostras foram confeccionadas com as hastes de aço carbono 1040, estas foram encapsulados em uma pasta de cimento tipo CP V-ARI (normalmente utilizado no encapsulamento das hastes de âncora, pela sua alta resistência mecânica inicial), preparada com uma relação entre água e cimento (a/c) de 0,5 , similar à de campo. Além desta amostra, considerada como referência (CIM-0), outras duas foram elaboradas pela contaminação artificial desta com teores adicionais de 2,0\% (CIM-2,0) e 3,5\% (CIM-3,5), em massa, de $\mathrm{NaCl}$, para a avaliação comparativa dos resultados. O pH resultante destas composições foi de 13,10 $\pm 0,05$.

As extremidades do cilindro de aço carbono foram isoladas do contato com o ambiente com fita auto fusão, de modo que uma área média de $(8,0 \pm 0,2) \mathrm{cm}^{2}$ na metade do cilindro ficou exposta para todos os sistemas em estudo. Este foi inserido pela tampa de um porta amostra de $350 \mathrm{~mL}$ com as marcações para cada eletrodo, como pode ser observado esquematicamente na Figura 2. Neste caso, esse material foi considerado o eletrodo de trabalho (ET) em uma célula de três eletrodos, com um contra eletrodo (CE) de grafite e um eletrodo de calomelano saturado (ECS) como referência (RE).

Todos os corpos de prova foram mantidos em cura úmida por $48 \mathrm{~h}$, para seu endurecimento, previamente à exposição em ambiente natural externo, por um período de tempo de 75 dias, em Curitiba, nas coordenadas $25^{\circ} 27^{\prime} 9^{\prime \prime} \mathrm{S}$ e $49^{\circ} 14^{\prime} 3^{\prime}$ 'O, em período de temperaturas diárias médias, variantes entre 5 a $23{ }^{\circ} \mathrm{C}$ e de umidade relativa superior a $80 \%$. As análises eletroquímicas foram realizadas ao longo desse intervalo.

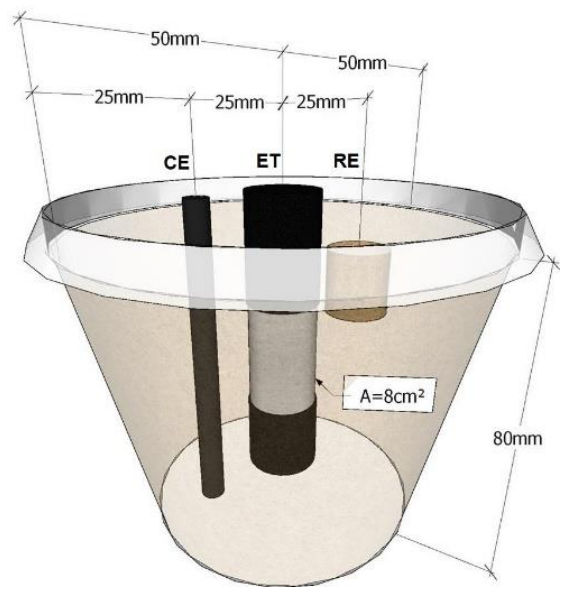

Figura 2: Desenho ilustrativo do sistema de medida de corrosão por EIE, em amostras cilíndricas de aço carbono 1040, encapsuladas, em solo e em pasta de cimento.

\subsection{Ensaios eletroquímicos}

As análises de EIE foram realizadas na célula eletroquímica montada com três eletrodos em potencial de circuito aberto (OCP), em solo e em pasta de cimento, ambas saturadas com água e durante o período de exposição natural em campo. Os ensaios foram conduzidos em uma faixa de frequência de $10 \mathrm{kHz}$ a $0,05 \mathrm{~Hz}$ com uma amplitude de sinal de $25 \mathrm{mV}$ (rms) com a aquisição de 7 pontos por década de frequência. Foi utilizado um potenciostato/galvanostato modelo PGSTAT 100 da marca AUTOLAB com um módulo FRA 2 para aquisição dos dados em laboratório, com temperatura ambiente controlada em $(23 \pm 2){ }^{\circ} \mathrm{C}$. O OCP foi registrado durante o período de exposição das amostras.

Os comportamentos eletroquímicos da amostra de aço em solo 01 e 02 foram avaliados, 6 h depois de conformado o sistema, aos 21 e aos 75 dias de exposição em ambiente natural. A variação do OCP foi acompanhada em 14, 21, 36, 58 e 75 dias.

As variações da impedância das amostras em pasta de cimento, CIM-0, CIM-2,0 e CIM-3,5, foram 
medidas às 48 h depois da cura úmida, em 21 e em 75 dias de exposição em ambiente natural externo. A variação do OCP com o tempo foi acompanhada nas seguintes idades: 48 h depois da cura úmida (inicial), 14, $21,36,58$ e 75 dias.

\subsection{Ensaio de caracterização dos produtos de corrosão}

As micrografias das superfícies dos metais foram obtidas em um microscópio eletrônico de varredura com emissão de elétrons por um canhão de emissão de campo (FEG-“Field Emission Gun”), modelo Mira3 LM, marca Tescan. As áreas para as análises foram obtidas a partir de um detector de elétrons secundários (ES). Também, foi verificada a composição química elementar da área exposta, com uma microssonda analítica de raios X, tipo EDS, marca Oxford. Os parâmetros do equipamento utilizados para a análise foram: a tensão de corrente do feixe de elétrons de $15 \mathrm{kV}$; a abertura do feixe de $18,0 \mathrm{~nm}$; a distância de trabalho de $15 \mathrm{~mm}$; e o modo de varredura por profundidade.

As análises de DRX foram realizadas nos produtos de corrosão retirados da superfície do metal exposto em solo, na forma de pó, em um equipamento da marca Bruker, modelo D8 ADVANCED ECO, com alvo de tubo de raios-X de cobre, $\lambda=154 \AA$.

\section{RESULTADOS}

Os comportamentos eletroquímicos das amostras de aço em solo 01 e solo 02 avaliados por EIE podem ser visualizados na Figura 3, a partir dos diagramas de Nyquist e do gráfico da variação do OCP com o tempo. Pela análise do gráfico, pode-se inferir por haver um eletrólito menos resistivo (solo 02) que o solo 01com o teor de íon cloreto contido no solo que houve variação dos valores de impedância de acordo com o teor do íon contaminante, sendo a impedância maior para o solo com menor teor de cloreto. Os valores de impedância diminuíram em função do tempo de exposição em ambiente natural externo. Com relação aos valores de OCP, houve uma tendência para valores menos negativos com o tempo de exposição.

Na Tabela 2, estão reunidos os respectivos valores de resistência da solução e de polarização extraídos dos diagramas de ambas as amostras.
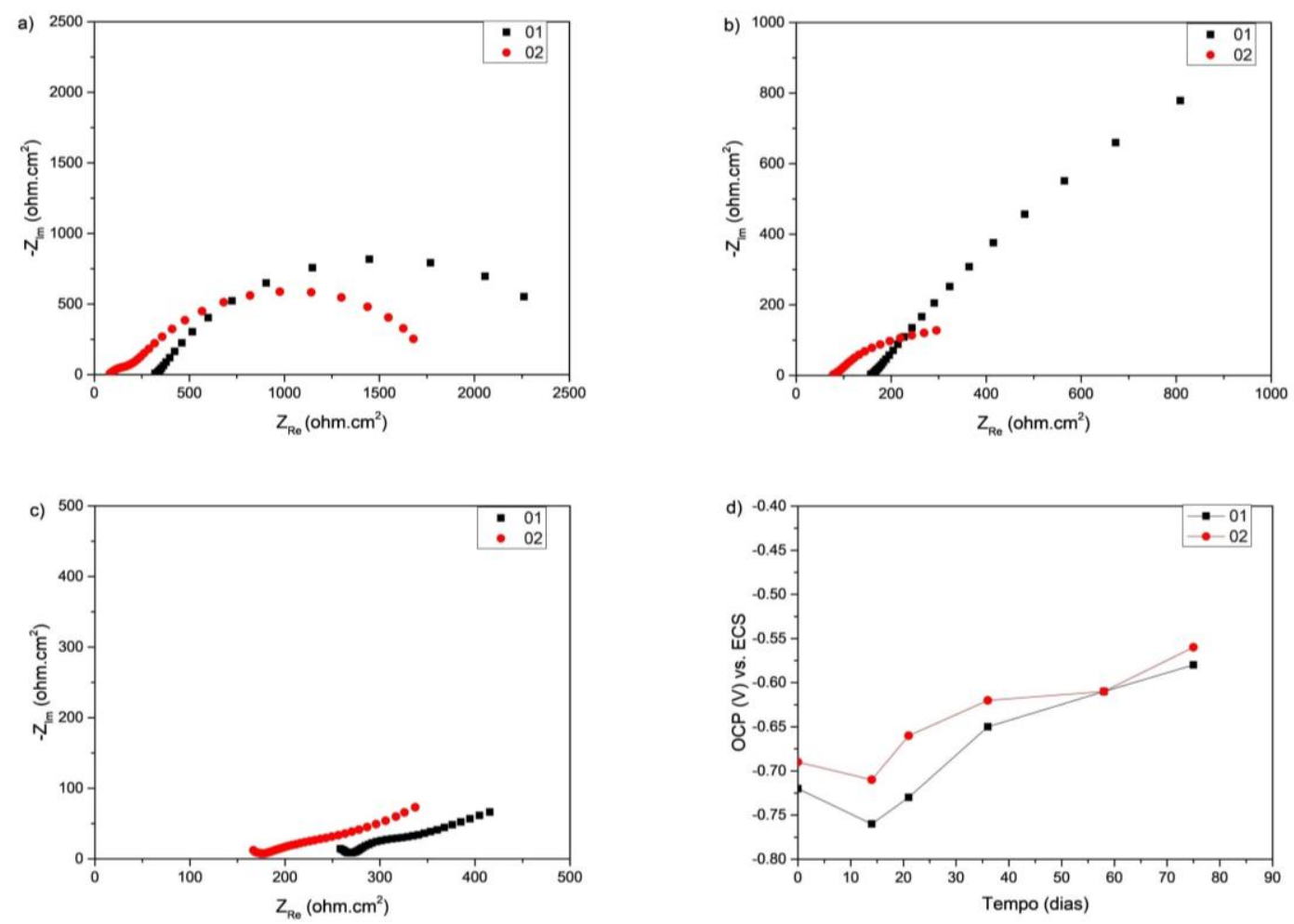

Figura 3: Diagramas de Nyquist e da variação do OCP para as amostras solo 01 e solo 02, sendo: a) Inicial, b) 21 dias c) 75 dias, d) OCP. 
Tabela 2: Valores de resistência da solução e de polarização das amostras solo 01 e solo 02 , obtidos às $6 \mathrm{~h}$ depois de conformadas as amostras (INICIAL), aos 21 e aos 75 dias de exposição dos sistemas em ambiente natural externo.

\begin{tabular}{|c|c|c|c|c|c|c|}
\hline AMOSTRA & $\begin{array}{l}R_{S} \quad\left(o h m . c m^{2}\right) \\
\text { INICIAL }\end{array}$ & $\begin{array}{l}\mathbf{R}_{\mathbf{P}}(\text { ohm.cm } \\
\text { INICIAL }\end{array}$ & $\begin{array}{l}R_{S}\left(\text { ohm.cm }{ }^{2}\right) \\
21 \text { DIAS }\end{array}$ & $\begin{array}{l}R_{P}\left(\text { ohm.cm }{ }^{2}\right) \\
21 \text { DIAS }\end{array}$ & $\begin{array}{l}R_{S}\left(\text { ohm.cm }{ }^{2}\right) \\
75 \text { DIAS }\end{array}$ & $\begin{array}{l}R_{P}\left(\text { ohm } . \mathrm{cm}^{2}\right) \\
75 \text { DIAS }\end{array}$ \\
\hline solo 01 & 345,3 & 2596,0 & 164,6 & 4742,4 & 267,0 & 111,8 \\
\hline solo 02 & 194,1 & 1641,1 & 79,8 & 501,6 & 164,5 & 294,1 \\
\hline
\end{tabular}

Os diagramas de Nyquist e do gráfico da variação do potencial de OCP com o tempo das amostras CIM-0, CIM-2,0 e CIM-3,5, estão apresentados na Figura 4. Os resultados de impedância inicial (depois de $48 \mathrm{~h}$ de cura úmida) e durante o envelhecimento até os 75 dias, apresentaram variação de acordo com os teores de cloreto na mistura, reduzindo em função do aumento do seu teor de CIM-0, CIM-2,0 a CIM-3,5. Os valores de OCP também foram similares em termos da concentração do íon cloreto na mistura (CIM-2,0 e CIM-3,5) e inferiores ao da amostra de referência CIM-0, e se mantiveram praticamente constantes com o tempo de exposição.

Os resultados de resistência a polarização e da solução das amostras CIM-0, CIM-2,0 e CIM-3,5, podem ser visualizados na Tabela 3 .
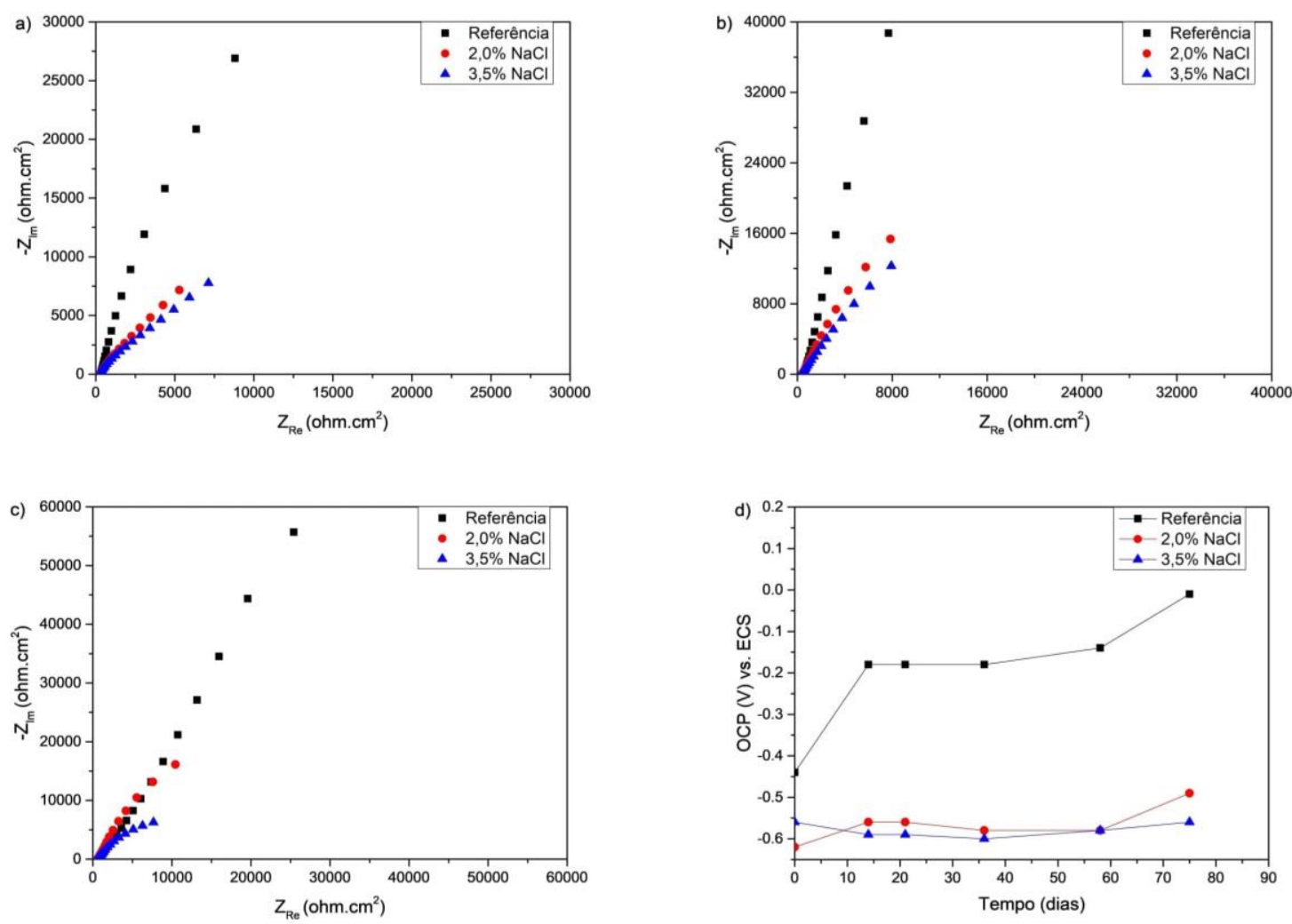

Figura 4: Diagramas Nyquist e variação do potencial de circuito aberto para o aço carbono encapsulado em pasta de cimento com diferentes teores de cloretos. a) Inicial (48 h), b) 21 dias c) 75 dias, d) OCP.

Tabela 3: Valores de resistência da solução e resistência a polarização do aço carbono na pasta de cimento, durante os 75 dias de exposição em ambiente natural.

\begin{tabular}{|c|c|c|c|c|c|c|}
\hline AMOSTRA & $\begin{array}{l}R_{\text {s }}(\text { ohm.cm } \\
\text { INICIAL }\end{array}$ & $\begin{array}{l}R_{p}\left(\text { ohm } . \mathrm{cm}^{2}\right) X \\
10^{4} \text {-INICIAL }\end{array}$ & $\begin{array}{l}R_{\mathrm{s}}\left(\text { ohm.cm }{ }^{2}\right) \\
21 \text { DIAS }\end{array}$ & $\begin{array}{l}R_{p}\left(\text { ohm } . \mathrm{cm}^{2}\right) X \\
10^{4}-21 \text { DIAS }\end{array}$ & $\begin{array}{l}R_{s}\left(\text { ohm.cm }{ }^{2}\right) \\
75 \text { DIAS }\end{array}$ & $\begin{array}{l}R_{p}\left(\text { ohm } . \mathrm{cm}^{2}\right) X \\
10^{4}-75 \text { DIAS }\end{array}$ \\
\hline CIM-0 & 280,6 & 21 & 669,0 & 140 & 1075,2 & 280 \\
\hline CIM-2,5 & 238,5 & 7,5 & 552,4 & 9,5 & 860,4 & 6,3 \\
\hline CIM-3,5 & 57,2 & 10 & 348,5 & 7,5 & 569,1 & 2,2 \\
\hline
\end{tabular}


Após 75 dias de exposição em ambiente natural externo, as amostras solo 01, solo 02, CIM-0, CIM2,0 e CIM-3,5 foram avaliadas superficialmente para a visualização da morfologia dos produtos de corrosão, primeiro, por inspeção visual e registro fotográfico, como mostrado na Figura 5 e, posteriormente, por MEV.

Diferentes características morfológicas foram encontradas nos produtos de corrosão na superfície do aço carbono inserido nas amostras, solo 01 e 02 , durante 75 dias. As micrografias da superfície do metal, por MEV podem ser visualizadas na Figura 6 (a-c) do solo 01 e Figura 6 (d-f) do solo 02. A composição química obtida pela análise semiquantitativa com o EDS, das regiões destacadas Figura 6 (a-f), estão disponíveis na Tabela 4.

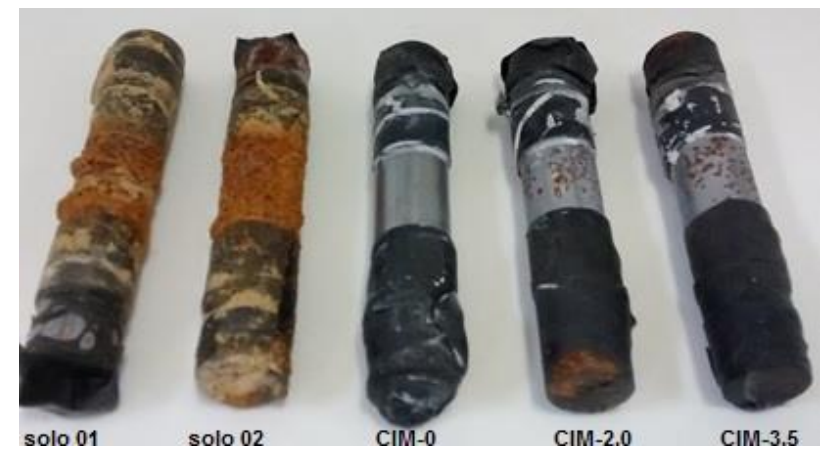

Figura 5: Foto referente às amostras solo 01 e 02, e CIM-0, CIM-2,0 e CIM-3,5, respectivamente, após um período de exposição de 75 dias em ambiente natural externo.
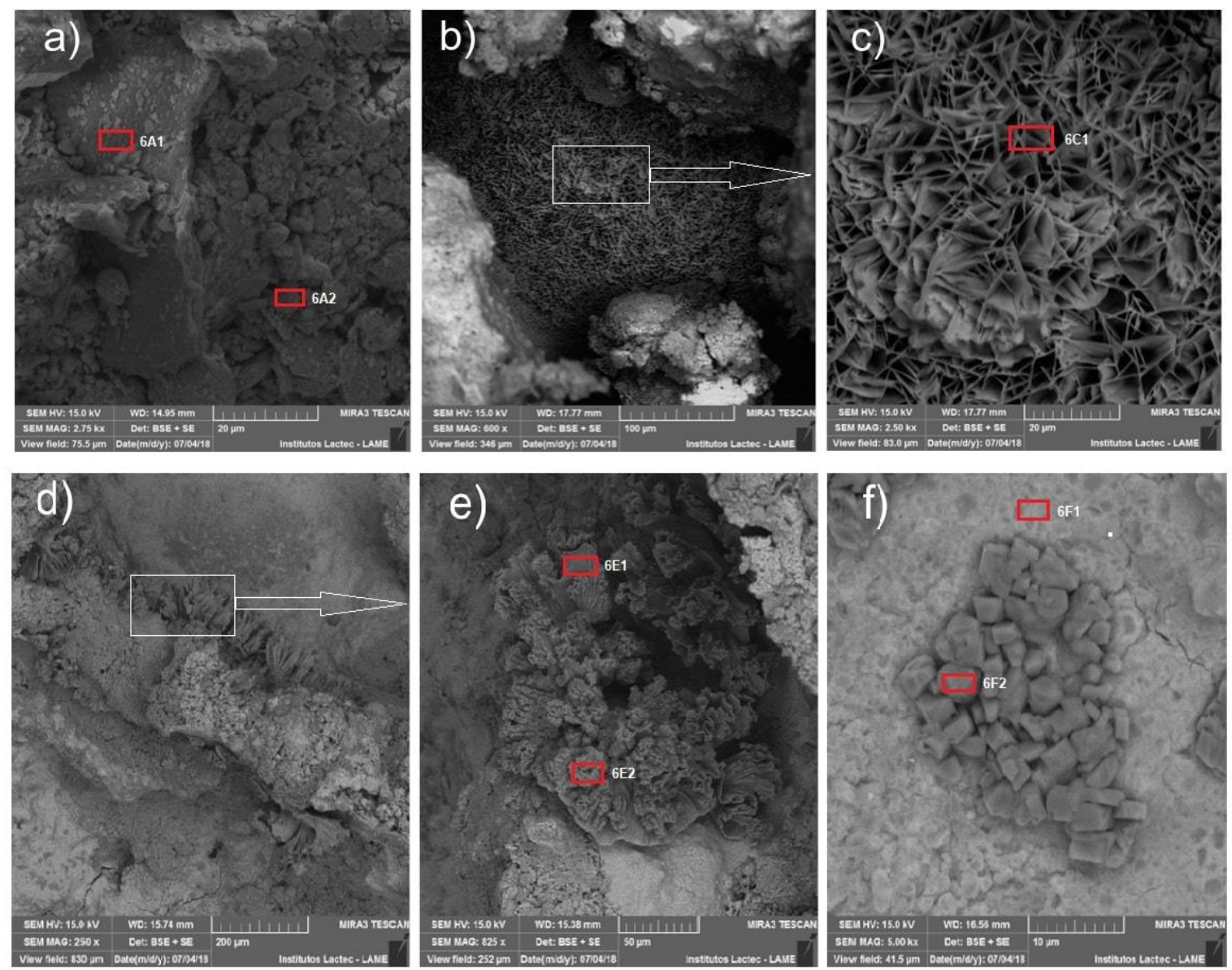

Figura 6: Imagens micrográficas, por MEV, dos produtos de corrosão encontrados na superfície do aço carbono imerso nas amostras solo 01 e 02 , depois dos 75 dias de exposição natural externa. 
Tabela 4: Composição química semiquantitativa elementar, em percentual em massa, encontrada nos produtos de corrosão das áreas em destaque na Figura 6 (a-f).

\section{ELEMENTOS QUÍMICOS (\% EM MASSA)}

\begin{tabular}{|c|c|c|c|c|c|c|c|c|c|}
\hline Área & $\mathrm{Fe}$ & $\mathrm{O}$ & $\mathrm{C}$ & $\mathrm{Ca}$ & $\mathrm{Al}$ & $\mathrm{Si}$ & $\mathrm{Na}$ & $\mathrm{K}$ & $\mathrm{Cl}$ \\
\hline $6 \mathrm{~A} 1$ & $65,1 \pm 0,6$ & $26,8 \pm 0,6$ & $1,3 \pm 0,4$ & - & - & $2,2 \pm 0,1$ & - & $1,9 \pm 0,1$ & - \\
\hline $6 \mathrm{~A} 2$ & $54,7 \pm 0,8$ & $30,3 \pm 0,7$ & - & - & $4,5 \pm 0,2$ & $8,5 \pm 0,3$ & - & $0,9 \pm 0,2$ & - \\
\hline $6 \mathrm{C} 1$ & $75,9 \pm 0,6$ & $22,9 \pm 0,6$ & - & - & $0,4 \pm 0,1$ & $0,7 \pm 0,1$ & - & - & - \\
\hline $6 \mathrm{E} 1$ & $1,2 \pm 0,4$ & $29,9 \pm 0,5$ & $0,6 \pm 0,1$ & $66,3 \pm 0,6$ & - & - & - & - & - \\
\hline $6 \mathrm{E} 2$ & $1,4 \pm 0,2$ & $34,6 \pm 0,3$ & $3,6 \pm 0,2$ & $59,3 \pm 0,3$ & - & - & - & - & - \\
\hline $6 \mathrm{~F} 1$ & $44 \pm 0,4$ & $38,6 \pm 0,5$ & $7,1 \pm 0,3$ & $0,8 \pm 0,1$ & $1,5 \pm 0,1$ & $3,4 \pm 0,1$ & $3,5 \pm 0,1$ & - & - \\
\hline $6 \mathrm{~F} 2$ & $1,3 \pm 0,1$ & $53,6 \pm 0,3$ & $15,5 \pm 0,2$ & $29,2 \pm 0,3$ & - & - & - & - & - \\
\hline
\end{tabular}

As análises dos produtos de corrosão por DRX indicaram a presença de goetita e calcita, além de quartzo e albita que são minerais encontrados no solo. Na Figura 7, foram identificados os produtos de corrosão encontrados na superfície do metal após 75 dias de exposição no solo.

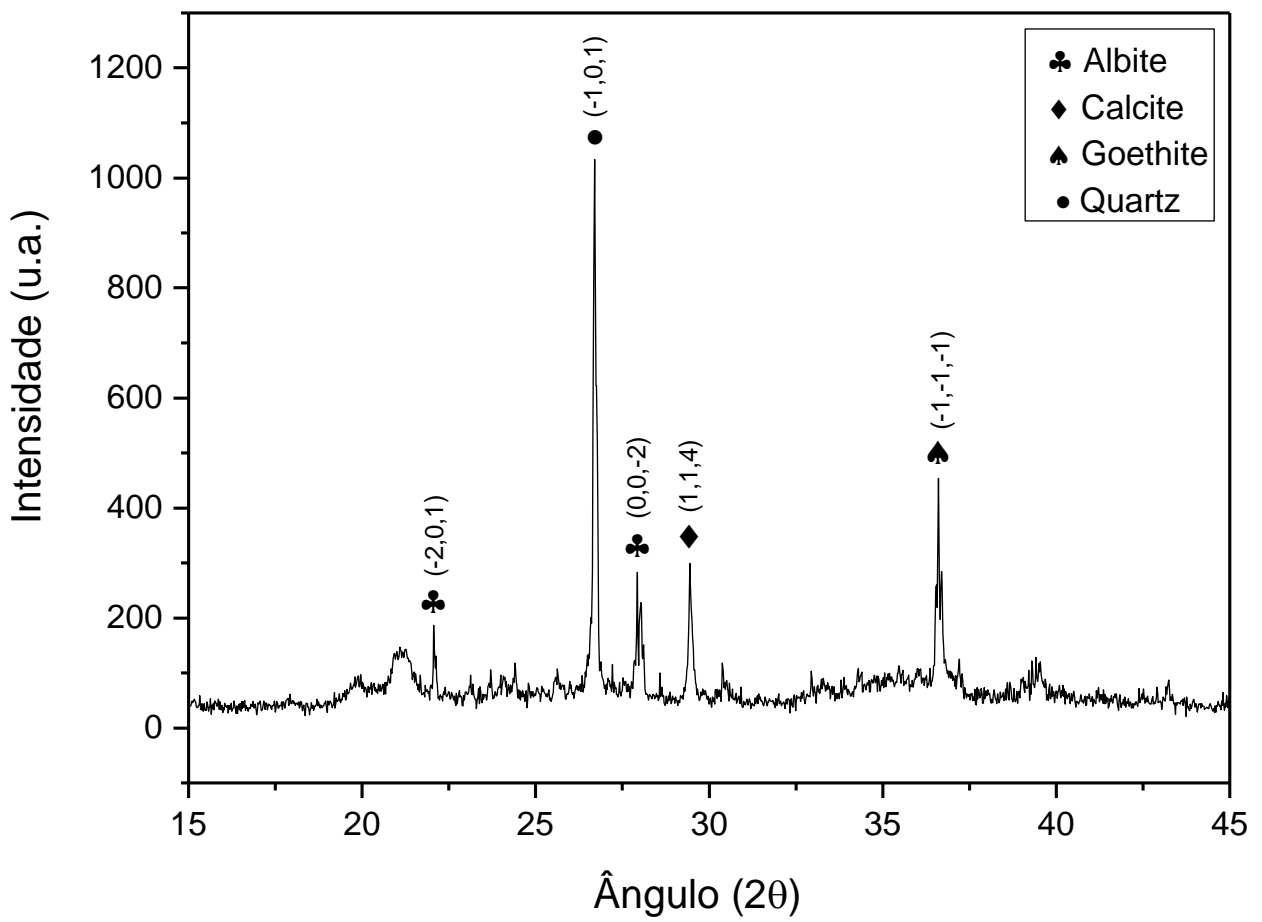

Figura 7: Difratograma de raios $X$ dos produtos de corrosão encontrados na superfície do metal imerso em solo por 75 dias.

Pela análise dos produtos de corrosão encontrados na superfície do aço carbono 1040, nas amostras CIM-2,0 e CIM-3,5, e os aspectos macroscópicos dos cilindros e imagens micrográficas por MEV, inferiu-se que os mesmos representaram uma corrosão localizada por pites, conforme apresentado na Figura 8 (a-d), amostra CIM-3,5, com diferentes ampliações.

Em destaque, nas imagens b e d, encontram-se apresentadas a superfície e a seção transversal do aço carbono contendo pites, respectivamente, e cujas profundidades de penetração e largura foram, aproximadamente, iguais e da ordem de $240 \mu \mathrm{m}$. Na Figura 8 (d), foi destacado, também, um segundo pite, cuja profundidade foi da ordem de $75 \mu \mathrm{m}$, e cerca de 2 vezes a sua largura superficial. 

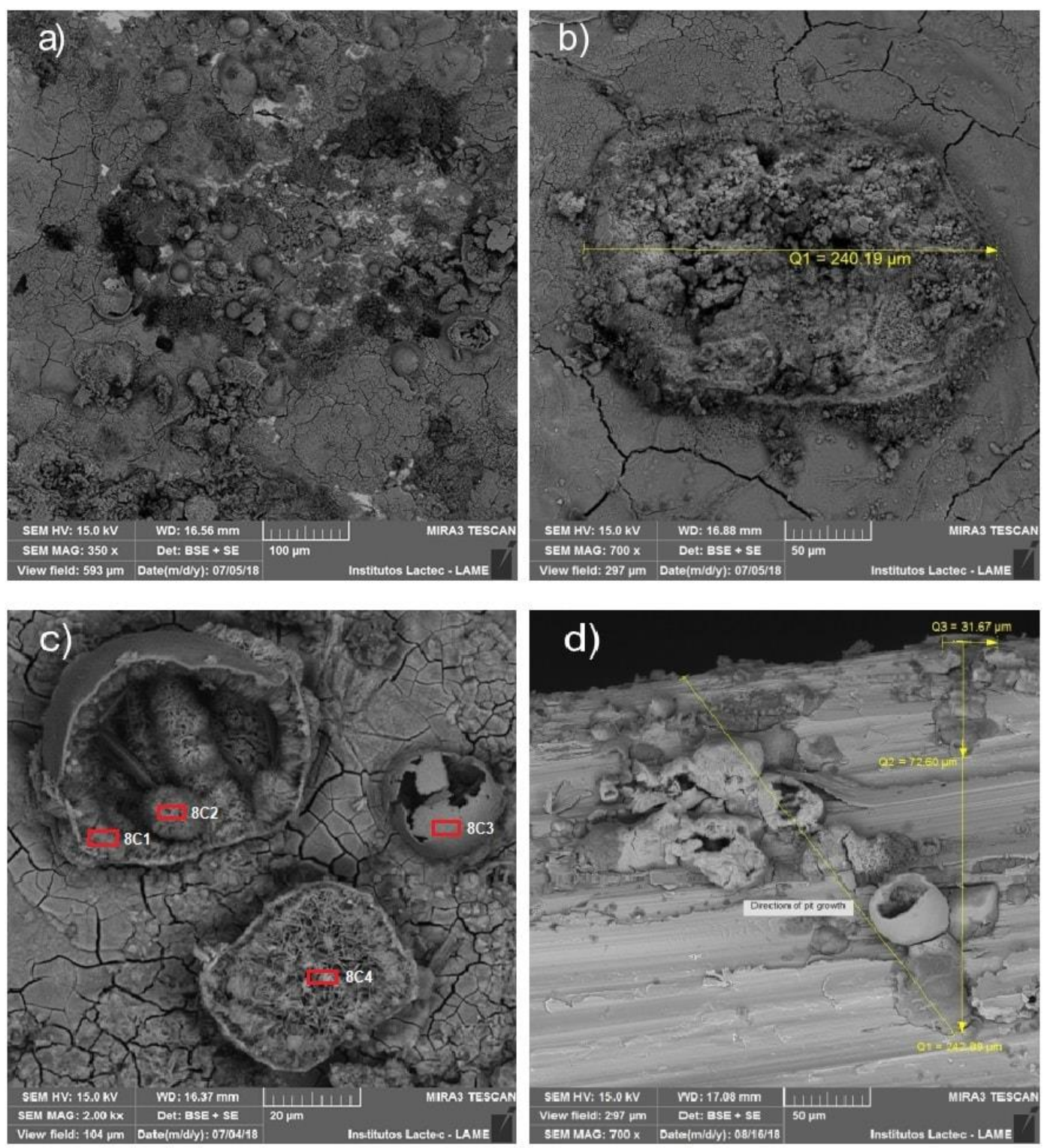

Figura 8: Imagens micrográficas, por $\mathrm{MEV}$, dos produtos de corrosão encontrados na superfície do aço carbono na amostra CIM-3,5, após 75 dias de exposição em ambiente natural externo com diferentes ampliações: a) 350X, b) 700X, c) $2000 \mathrm{X}, \mathrm{d}) 700 \mathrm{X}$.

Os resultados da composição química elementar semiquantitativa nos produtos de corrosão da Figura 8(c) está apresentado na Tabela 5, em função de que há diferentes características morfológicas em um mesmo local.

Tabela 5: Composição química elementar semiquantitativa encontrada nos produtos de corrosão da superfície do aço carbono 1040, nas áreas em destaque na Figura 8.

\begin{tabular}{l|l|l|l|l|l|l|l|l|l}
\hline \multicolumn{1}{c}{ ELEMENTOS QUímICOS (\% EM MASSA) - AMOSTRA CIM-3,5 } \\
\hline Área & $\mathrm{Fe}$ & $\mathrm{O}$ & $\mathrm{C}$ & $\mathrm{Ca}$ & $\mathrm{Cl}$ & $\mathrm{Si}$ & $\mathrm{Na}$ & $\mathrm{K}$ & $\mathrm{Al}$ \\
\hline $8 \mathrm{C} 1$ & $54,1 \pm 0,7$ & $33,4 \pm 0,7$ & $5,9 \pm 0,5$ & $2,0 \pm 0,1$ & $3,2 \pm 0,1$ & - & - & - & \\
\hline $8 \mathrm{C} 2$ & $62,4 \pm 0,5$ & $28,0 \pm 0,5$ & $3,3 \pm 0,3$ & $1,4 \pm 0,1$ & $3,7 \pm 0,1$ & - & - & - & \\
\hline $8 \mathrm{C} 3$ & $71,1 \pm 0,4$ & $21,4 \pm 0,4$ & - & $0,8 \pm 0,1$ & $6,2 \pm 0,1$ & - & - & - & \\
\hline $8 \mathrm{C} 4$ & $71,0 \pm 0,4$ & $21,6 \pm 0,4$ & - & $0,7 \pm 0,1$ & $7,7 \pm 0,2$ & - & - & - & \\
\hline
\end{tabular}




\section{DISCUSSÃO}

A corrosão do aço carbono, induzida por cloretos, ocorreu de maneira diferente no solo e na pasta de cimento. Enquanto no solo o aço foi corroído de forma generalizada, na pasta de cimento a corrosão localizada ficou evidente, até mesmo visualmente, conforme mostrado na Figura 5, sendo esta, corroborada com as imagens micrográficas por MEV, mostrada em detalhe na Figura 8 (c-d).

Pela análise dos diagramas de Nyquist com as medidas de impedância eletroquímica do aço carbono 1040, foi possível visualizar as alterações nos seus valores de $R_{s}$ e $R_{p}$, bem como do OCP, em função do tempo de exposição em ambiente natural externo, tanto no solo quanto na pasta de cimento. A influência do teor de cloretos na velocidade de formação dos produtos de corrosão foi verificada pela redução do semicírculo no diagrama de Nyquist, acompanhada da redução da resistência a polarização do metal, nas amostras solo 01 e 02 e em CIM-2,0 e CIM-3,5. Já, na amostra solo 01, com um menor teor de cloretos, este, naturalmente presente no solo retirado do entorno de uma das torres de transmissão em estudo de caso, ocorreu o aumento da $\mathrm{R}_{\mathrm{p}}$ até os 21 dias, como pode ser visualizado na Tabela 2.

Após os 75 dias de exposição em ambiente natural externo, os diagramas de Nyquist das amostras solo 01 e 02 , tiveram comparativamente formatos semelhantes, entretanto, foram obtidas diferenças nos valores de $R_{p}$ e, também, de $R_{s}$, este último, como função da maior concentração iônica na solução aquosa do meio, portanto, sendo menor para a amostra solo 02 . Já, para o $\mathrm{R}_{\mathrm{p}}$, considerou-se haver influência no resultado a formação de um maior volume de produtos de corrosão, detectada visualmente, na superfície da amostra solo 02 , ocasionando, consequentemente, uma maior resistência complexa à passagem da corrente alternada aplicada, após 75 dias, o que corroborou com os resultados de análises inferidos na literatura [23].

Na pasta de cimento, os valores de $R_{p}$ foram elevados em relação ao do solo (cerca de $10^{4}$ vezes maior), como foi possível visualizar pelo diagrama de Nyquist e os valores calculados, na Figura 4 e Tabela 3 , respectivamente. Na amostra CIM-0, observou-se o aumento da resistência à polarização durante os 75 dias de exposição, enquanto na amostra CIM-2,0 ocorreu um aumento de $\mathrm{R}_{\mathrm{p}}$ até os 21 dias e no período seguinte, até os 75 dias, houve uma redução no valor observado. Por outro lado, a redução nos valores de $R_{p}$ foi constatada na amostra CIM-3,5, durante os 75 dias de exposição, o que evidenciou a influência do teor de cloretos no processo corrosivo [24,25].

As medidas de OCP nas amostras solo 01 e 02 , evidenciaram um deslocamento para valores menos negativos com o tempo de exposição, devido à corrosão do metal e formação de uma barreira de produtos de corrosão. Esse deslocamento nos valores de OCP foi acompanhado da redução dos valores de impedância como pode ser visualizado na Figura 3 e Tabela 2.

Nas amostras CIM-2,0 e CIM-3,5 os valores de OCP mantiveram-se ao redor de $-0,55$ e de $-0,60 \mathrm{~V}$, sendo que uma tendência para os potenciais mais positivos foi observada na amostra CIM- 0 , como pode ser visualizado na Figura 4 (d). Esses resultados estão de acordo com os encontrados na literatura, onde os potenciais mais negativos indicam uma maior probabilidade à corrosão do metal em concreto e os potenciais mais positivos os de menor probabilidade [26]. Segundo a norma da ASTM C876 [27] os valores de potencial mais negativos que $-0,35 \mathrm{~V}$ em relação ao eletrodo de referência de prata cloreto de prata, indicam uma probabilidade de mais de $90 \%$ de estar ocorrendo corrosão em um metal, e para valores mais positivos que $-0,20 \mathrm{~V}$ de não estar ocorrendo corrosão do metal no concreto, o que corrobora com os resultados obtidos. Apesar do ECS ter sido utilizado na obtenção dos dados nesse trabalho a diferença de potencial em relação ao eletrodo de referência de prata cloreto de prata saturado com cloreto de potássio é de apenas $0,02 \mathrm{~V}$ [28].

As características morfológicas dos produtos de corrosão encontrados no aço carbono em solo, Figura 6 (a-f) foram diversificadas, devido sobretudo à complexa composição do solo. Na Figura 6 (a) foi possível observar uma placa compacta que possivelmente pode ser de magnetita (dada a sua composição elementar semiquantitativa de $72 \% \mathrm{Fe}, 28 \% \mathrm{O}$ ) formada no início do processo corrosivo e que foi parcialmente transformada em outros produtos de corrosão, o que está em concordância com os resultados obtidos pelos espectros de EDS presentes na Tabela 4, e os diversos estudos sobre estruturas de produtos de corrosão em ambientes atmosféricos presentes na literatura [29,30,31]. As estruturas na forma de pétalas encontradas na Figura 6 (b) (em destaque 6 (c)) foram atribuídas à lepidocrocita e ou goetita, e foram encontradas em estágios iniciais de corrosão, confinadas em um poro onde outros produtos de corrosão foram visualizados nas paredes destes, corroborando com alguns resultados encontrados na literatura [32,33]. A composição por EDS da área em destaque confirmou uma tendência da presença de oxi-hidróxidos de ferro como a $\gamma$ - $\mathrm{FeOOH}$ e $\alpha-\mathrm{FeOOH}$, feita por cálculos estequiométricos. A análise de DRX, Figura 7, confirmou a presença de goetita $(\alpha-\mathrm{FeOOH})$, entre outros compostos, nos produtos de corrosão. Entretanto, a lepidocrocita não foi detectada. Wang et al. [3], destacou em sua pesquisa que estes produtos de corrosão podem se transformar em goetita ao longo do tempo, pois esta é considerada um composto mais estável. 
Em outras regiões das amostras, solo 01 e solo 02, foram encontrados produtos de corrosão na forma de "flores", Figura 6_(d) (em destaque Figura 6 (e)), junto com outros produtos de corrosão do aço carbono. Essas formações foram constituídas por Ca, C e O (Tabela 4), atribuindo-se por cálculos estequiométricos constituir de hidróxidos, carbonatos e óxidos de cálcio. $\mathrm{O}$ pH do solo na região de análise foi de $(9,42 \pm 0,05)$. Em uma região próxima dessas formações foi possível observar, Figura 6 (f), a presença de cristais de $\mathrm{CaCO}_{3}$ que podem ser os precursores para a formação dessas estruturas na forma de "flores", conforme já identificada por DRX, na Figura 7.

A ocorrência de corrosão localizada no aço carbono em pasta de cimento foi observada para as amostras CIM-2,0 e CIM-3,5. Na Figura 8 (c) (ampliação das imagens 7 (a) e 7(b)), foi possível observar os diferentes pites formados, onde a área próxima ao local destacado $8 \mathrm{C} 3$ pode ser correspondente à quebra da barreira do óxido protetor $\left(\mathrm{Fe}_{3} \mathrm{O}_{4}\right.$ ou $\left.\mathrm{Fe}_{2} \mathrm{O}_{3}\right)$, como corroborado pela composição química por EDS da superfície dessa região, Tabela 5, que possui, além do óxido, um alto teor de cloretos $(6,2 \%)$, em relação ao teor do contaminante adicionado, adsorvido a sua superfície. Em uma região próxima, 8C4, foi possível visualizar um pite onde foi encontrada uma concentração ainda maior de cloretos na superfície $(7,7 \%)$. Por fim, na área com maior formação de pites, na Figura 8 (c) (área destacada 8C1 e 8C2), foi possível visualizar alguns produtos formados na borda e dentro de um destes. As imagens dos pites estão de acordo com os trabalhos encontrados na literatura, onde mesmo em estágios iniciais de cura da pasta de cimento contaminada com cloreto, quando o aço se encontraria no estado passivo, a corrosão localizada pode ocorrer $[34,35,36]$.

\section{CONCLUSÕES}

O processo analítico da verificação do desempenho quanto à corrosão do aço carbono 1040 imerso em solo e embutido em pasta de cimento, foi evidenciado pela EIE, a partir dos cálculos de $\mathrm{R}_{\mathrm{p}}$ no diagrama de Nyquist, e, também, pela variação do OCP nestes sistemas, durante os 75 dias de suas exposições em ambiente natural externo. $\mathrm{O}$ valor comparativo de OCP, medido ao longo do tempo de exposição ao ambiente natural externo, mostrou ser um parâmetro de análise do processo corrosivo, servindo como indicador do estado passivo e ativo do aço carbono 1040 na pasta de cimento

Como resultado das técnicas adotadas, pode-se inferir que o processo de corrosão nos corpos de prova do metal foi mais evolutivo em função do maior teor de contaminante nas amostras e que as características morfológicas dos produtos de corrosão foram diversificadas em solo e meio cimentício,

Por fim, as conclusões deste trabalho podem auxiliar na compreensão, do processo de corrosão do sistema de ancoragem de torres estaiadas de linha de transmissão de energia elétrica, a influência da agressividade do solo no processo corrosivo, bem como na seleção de materiais com maior durabilidade, aumentando a confiabilidade e a vida útil dessas estruturas.

\section{AGRADECIMENTOS}

Os autores agradecem à infraestrutura e ao apoio em recursos humanos e financiamento à TAESA, projeto P\&D 5012-0045/2016, à ANEEL, aos Institutos LACTEC, ao CNPq Lei 8010/90 (LI 15/2187214-1; LI 14/4695814-5; LI14/3410726-9), ao P\&D COPEL 6491-0301/2013, ao CNPq/PIBIT e de bolsa DT, processo 302672/2016-8, e à Universidade Federal do Paraná - PIPE/UFPR.

\section{BIBLIOGRAFIA}

[1] KRISHNADEV, M., LAROUCHE, M., LAKSHMANAN, V.I., et al.,"Fracture of high carbon high strength anchor rods”, Engineering Failure Analysis, v. 15, n. 8, pp. 1009-1018, 2008.

[2] RIEDER, E.S., BRUSAMARELLO, V., BIANCHI, A.L., et al., "Investigação dos principais processos de corrosão em estações de energia elétrica do Estado do RS”, Matéria, v. 14, n. 3, pp. 1000-1014, 2009.

[3] WANG, S., DU, C., LI, X., et al., "Field corrosion characterization of soil corrosion of X70 pipeline steel in a red clay soil”, Progress in Natural Science: Materials International, v. 25, n. 3, pp. 242-250, 2015.

[4] LI, S., JUNG, S., PARK, K., LEE, S.-M., et al., "Kinetic study on corrosion of steel in soil environments using electrical resistance sensor technique", Materials Chemistry and Physics, v. 103, n. 1, pp. 9-13, 2007.

[5] YAN, M., SUN, C., DONG, J., et al., "Electrochemical investigation on steel corrosion in iron-rich clay", Corrosion Science., v. 97, pp. 62-73, 2015.

[6] ISMAIL, A.I.M., EL-SHAMY, A.M., "Engineering behaviour of soil materials on the corrosion of mild steel”, Applied Clay Science, v. 42, n. 3-4, pp. 356-362, 2009.

[7] MONTOYA, P., MARÍN, T.,ECHAVARRÍA, A., CALDERÓN, J.A., "Influence of anion and pH on the 
electrochemical co-deposition and transformation of Iron oxy-hydroxide", International Journal of Electrochemical Science, v. 8, n. 12, pp. 12566-12579, 2013.

[8] YAN, M., SUN, C., XU, J., et al., "Role of Fe oxides in corrosion of pipeline steel in a red clay soil", Corrosion Science, v. 80, pp. 309-317, 2014.

[9] WU, T., XU, J., SUN, C., et al., "Microbiological corrosion of pipeline steel under yield stress in soil environment", Corrosion Science, v. 88, pp. 291-305, 2014.

[10] KOSEC, T., LEGAT, A., BOKAN-BOSILJKOV, V., "Corrosion Properties of Different Forms of Carbon Steel in Simulated Concrete Pore Water", Materials and technology, v. 48, n. 1, pp. 51-57, 2014.

[11] DUFFÓ, G.S., MORRIS, W., RASPINI, I., et al., "A study of steel rebars embedded in concrete during 65 years", Corrosion Science, v. 46, n. 9, pp. 2143-2157, 2004.

[12] POURBAIX, M., "Thermodynamics and corrosion - a lecture”, Corrosion Science, v. 30, n. 10, pp. $963-$ $988,1990$.

[13] ROVENTI, G., BELLEZZE, T., GIULIANI, G., et al., "Corrosion resistance of galvanized steel reinforcements in carbonated concrete: Effect of wet-dry cycles in tap water and in chloride solution on the passivating layer", Cement and Concrete Reseach, v. 65, pp. 76-84, 2014.

[14] ANN, K.Y., SONG, H.W., "Chloride threshold level for corrosion of steel in concrete", Corrosion Science, v. 49, n. 11, pp. 4113-4133, 2007.

[15] XU, J., JIANG, L., WANG, J., "Influence of detection methods on chloride threshold value for the corrosion of steel reinforcement", Construction and Building Materials, v. 23, n. 5, pp. 1902-1908, 2009.

[16] ANGST, U.M., ELSENER, B., LARSEN, C.K., et al., "Critical chloride content in reinforced concrete A review", Cement and Concrete Research, v. 39, n. 12, pp. 1122-1138, 2009.

[17] DEUS, J.M., DÍAZ, B., FREIRE, L., et al., "The electrochemical behaviour of steel rebars in concrete: An Electrochemical Impedance Spectroscopy study of the effect of temperature", Electrochimca Acta, v. 131, 2014.

[18] BARBALAT, M., LANARDE, L., CARON, D., et al., "Electrochemical study of the corrosion rate of carbon steel in soil: Evolution with time and determination of residual corrosion rates under cathodic protection", Corrosion Science, v. 55, pp. 246-253, 2012.

[19] LASIA, A., Electrochemical Impedance Spectroscopy and its Applications, 1 ed., New York, Springer, 2014.

[20] CHAKRI, S., FRATEUR, I., ORAZEM, M.E., et al., "Improved EIS Analysis of the Electrochemical Behaviour of Carbon Steel in Alkaline Solution”, Electrochimca Acta, v. 246, pp. 924-930, 2017.

[21] SANTOS, M.C., PINHEIRO, C.L., DE FILHO, J. C. A. C., "Investigação do processo de corrosão causado pela polpa de bauxita em mineroduto de aço carbono", Matéria, v. 20, n. 1, pp. 178-184, 2015.

[22] MATOS, T. S., PORTELLA, K. F., BRAGANCA, M. O. G. P., et al., "Effect of chloride-induced corrosion on the mechanical properties of galvanized carbon steel anchor rods of guyed power transmission line", Espaço Energia, v. 28, pp. 20-25, 2018.

[23] KREYSA, G., Encyclopedia of Applied Electrochemistry, 1 ed., New York, Springer Reference, 2014.

[24] HORNBOSTEL, K., ANGST, U.M., ELSENER, B., et al., "Influence of mortar resistivity on the ratelimiting step of chloride-induced macro-cell corrosion of reinforcing steel", Corrosion Science, v. 110, pp. 46-56, 2015.

[25] ANGST, U.M., ELSENER, B., LARSEN, C.K., et al., "Chloride induced reinforcement corrosion: Electrochemical monitoring of initiation stage and chloride threshold values", Corrosion Science, v. 53, n. 4, pp. 1451-1464, 2011.

[26] POURSAEE, A., Corrosion of steel in concrete structures, 1 ed., New York, Elsevier, 2016.

[27] ASTM INTERNATIONAL, "Standard C876 - Standard test method for corrosion potentials of uncoated reinforcing steel in concrete", ASTM International, pp. 1-8, 2015.

[28] POPOV, B.N., Corrosion Engineering Principle and Solved Problems, 1 ed., New York, Elsevier, 2015.

[29] ALCÁNTARA, J., DE LA FUENTE, D., CHICO, B., et al., "Marine atmospheric corrosion of carbon steel: A review", Materials, v. 10, n. 4, 2017.

[30] ALCÁNTARA, J., CHICO, B., DÍAZ, I., et al., "Airborne chloride deposit and its effect on marine atmospheric corrosion of mild steel”, Corrosion Science, v. 97, pp. 74-88, 2015. 
[31] ALCÁNTARA, J., CHICO, B., SIMANCAS, J., et al., "An attempt to classify the morphologies presented by different rust phases formed during the exposure of carbon steel to marine atmospheres", Materials Characterization, v. 118, pp. 65-78, 2016.

[32] DUFFÓ, G.S., REINOSO, M., RAMOS, C.P., et al., "Characterization of steel rebars embedded in a 70year old concrete structure", Cement and Concrete Research, v. 42, n. 1, pp. 111-117, 2012.

[33] RAMAN, A., NASRAZADANI, S., SHARMA, L., "Morphology of rust phases formed on weathering steels in various laboratory corrosion tests", Metallography, v. 22, n. 1, pp. 79-96, 1989.

[34] APOSTOLOPOULOS, C.A., DEMIS, S., PAPADAKIS, V.G., "Chloride-induced corrosion of steel reinforcement - Mechanical performance and pit depth analysis", Construction and Building Materials, v. 38, pp. 139-146, 2013.

[35] DARMAWAN, M.S., "Pitting corrosion model for reinforced concrete structures in a chloride environment", Magazine of Concrete Research, v. 62, n. 2, pp. 91-101, 2010.

[36] SOLTIS, J., "Passivity breakdown, pit initiation and propagation of pits in metallic materials - Review", Corrosion Science, v. 90, pp. 5-22, 2015.

\section{ORCID}

Tiago Scheffer de Matos

https://orcid.org/0000-0002-6383-1835

Kleber FranKe Portella

http://orcid.org/0000-0002-0631-9990

Sérgio Luiz Henke

http://orcid.org/0000-0002-4322-0930

Mariana D’Orey Gaivão Portella Bragança

https://orcid.org/0000-0002-2702-7196

Igor Miguel Pinkoski

https://orcid.org/0000-0002-8682-622X

Alessandro Cesar de Sousa Berrêdo

https://orcid.org/0000-0002-4541-7809 